\title{
Suppressing Activity of an Array of Coupled Fitzhugh-Nagumo Oscillators
}

\author{
E. Adomaitiené $\dot{a}^{a}$, G. Mykolaitis ${ }^{b}$, S. Bumeliené ${ }^{a}$ And A. TAmaševiČius ${ }^{a, *}$ \\ ${ }^{a}$ Department of Electronics, Center for Physical Sciences and Technology, LT-01108 Vilnius, Lithuania \\ ${ }^{b}$ Department of Physics, Vilnius Gediminas Technical University, LT-10223 Vilnius, Lithuania
}

\begin{abstract}
An extremely simple method for stabilizing unstable steady states in an array of coupled neuronal FitzHughNagumo type oscillators is described. A two-terminal electronic feedback controller has been developed. The feedback circuit, when coupled to an array of oscillators, damps the spiking neurons, thus does away with the effect of synchronization. Both, numerical simulations and hardware experiments with the electronic circuits have been performed. The results for an array of three mean-field coupled FitzHugh-Nagumo oscillators are presented.
\end{abstract}

DOI: 10.12693/APhysPolA.129.562

PACS/topics: 05.45.Xt, 87.19.lr

\section{Introduction}

Synchronization of coupled oscillators is a common observation in a variety of fields in nature, science and engineering [1]. The phenomenon has been widely investigated in physical, electronic, chemical, and biological systems, where it has been found to occasionally can give rise to rather surprising effects. For example, too strong synchronization of neurons in the brain can end up in the Parkinson disease symptoms. The standard therapy for patients is electrical deep brain stimulation (DBS) with strong relatively high repetition rate $(\approx 100 \mathrm{~Hz})$ pulse trains, which damp the spiking neurons. Unfortunately, this treatment is often accompanied with side effects. A large number of more sophisticated feedback and non-feedback techniques to avoid synchronization of interacting oscillators in general, and more specifically with the possible application to neuronal arrays, have been described in literature [2-9].

The main purpose of the present research is to develop an alternative control method and to present its electronic implementation for damping the FitzHughNagumo (FHN) type neuronal oscillators, more specifically for stabilizing their unstable steady states.

\section{Array of coupled oscillators and feedback controller}

The general setup for damping the oscillations in a neuronal array is sketched in Fig. 1a. Here we consider a small array, composed of only three oscillators O1, O2, and O3. However, the analysis can be extended to larger arrays as well. The $\mathrm{CN}$ is a coupling node, in general, not accessible from the outside directly, but via some series resistor $R_{\mathrm{g}}$. The $\mathrm{DN}$ is an accessible damping node.

*corresponding author; e-mail: arunas.tamasevicius@ftmc.lt
The FC is the electronic feedback controller. The circuit diagram of the electronic feedback controller FC is shown in Fig. 1b.

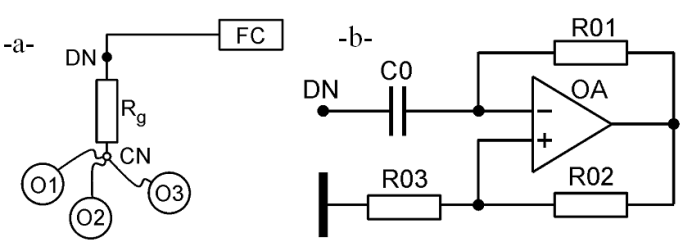

Fig. 1. (a) Block diagram of coupled oscillators with a feedback controller, (b) circuit diagram of the feedback controller.

We emphasize that the controller FC is an essentially two-terminal device. OA is a general-purpose operational amplifier, e.g. NE5534 type device. $C_{0}$ is an adjustable value of the capacitor; should be selected to ensure $R *$ $C_{0} \gg T / 2 \pi$ (here $T$ is the spike period). $R_{01}=R_{02}=$ $1 \mathrm{k} \Omega, R_{03}$ is an adjustable resistor to fit approximately the $R_{\mathrm{g}}$.

The individual oscillators $\mathrm{O}_{i}(i=1,2,3)$ are very similar to the electrical circuit, described in [10]. Our modified circuit is sketched in Fig. 2. The OA is a generalpurpose operational amplifier. The diodes D1 and D2 are the Schottky devices with the forward voltage drop $V^{*}$ of about $0.2 \mathrm{~V}$ at $1 \mathrm{~mA}$. Other circuit element values are the following: $L=100 \mathrm{mH}, C=22 \mathrm{nF}, R_{1}=R_{2}=1 \mathrm{k} \Omega$, $R_{3}=560 \Omega, R_{4}=30 \Omega, R_{5}=470 \Omega, R_{6}=220 \Omega$, $R_{7}^{(1)}=47 \mathrm{k} \Omega, R_{7}^{(2)}=49 \mathrm{k} \Omega, R_{7}^{(3)}=52 \mathrm{k} \Omega, R^{*}=470 \Omega$, $V_{0}=-15 \mathrm{~V}$. We note that the resistors $R_{7}^{(1,2,3)}$ are intentionally set slightly different in each individual oscillator to make them non-identical units. The main difference between the circuit in [10] and in the present circuit (Fig. 2) is, that in the LC tank the inductance $L$ is decreased from $1 \mathrm{H}$ to $100 \mathrm{mH}$ and the capacitance $C$ is reduced from $330 \mathrm{nF}$ to $22 \mathrm{nF}$ in order to move the operating frequency of the oscillators into the $\mathrm{kHz}$ range and to make the recording of the signals more convenient. 


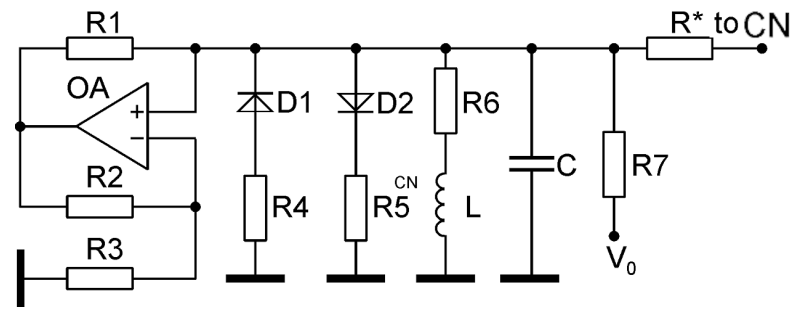

Fig. 2. Circuit diagram of the FHN type oscillator.

\section{Differential equations}

Using the Kirchhoff laws and introducing the following set of dimensionless variables and parameters

$$
\begin{aligned}
& x_{i}=\frac{V_{C}^{(i)}}{V^{*}}, \quad y_{i}=\frac{\rho I_{L}^{(i)}}{V^{*}}, \quad z=\frac{V_{C 0}}{V^{*}}, \quad \theta=\frac{t}{\tau}, \\
& \left\langle x_{i}\right\rangle=\frac{1}{N} \sum_{i=1}^{N} x_{i}, \quad \rho=\sqrt{\frac{L}{C}}, \quad \tau=\sqrt{L C}, \\
& a=\frac{\rho\left(R_{7}-R_{3}\right)}{R_{3} R_{7}}, \quad b=\frac{R_{6}}{\rho}, \quad c_{i}=\frac{\rho}{R_{7}^{(i)}} \frac{V_{0}}{V^{*}}, \\
& d_{1}=\frac{\rho}{R_{4}}, \quad d_{2}=\frac{\rho}{R_{5}}, \quad k=\frac{\rho}{R^{*}}, \quad \varepsilon=\frac{C}{C_{0}},
\end{aligned}
$$

also an asymmetric nonlinear function (different from the common FHN cubic function $\left.x^{3}[11]\right)$ :

$$
f(x)=\left\{\begin{array}{cc}
d_{1}(x+1), & x<-1, \\
0, & -1 \leq x \leq 1, \\
d_{2}(x-1), & x>1,
\end{array}\right.
$$

where the slope parameters are essentially different $\left(d_{1} \gg d_{2}\right)$, we arrive to the two sets of differential equations, convenient for numerical integration. The first set of $2 N$ differential equations

$$
\begin{aligned}
& \dot{x}_{i}=a x_{i}-f\left(x_{i}\right)-y_{i}+c_{i}+k\left(\left\langle x_{i}\right\rangle-x_{i}\right), \\
& \dot{y}_{i}=x_{i}-b y_{i}, \quad i=1,2, \ldots N
\end{aligned}
$$

is valid in the case of an uncontrolled array, whereas the set of $2 N+1$ differential equations

$$
\begin{aligned}
& \dot{x}_{i}=a x_{i}-f\left(x_{i}\right)-y_{i}+c_{i}+k\left(z-x_{i}\right), \\
& \dot{y}_{i}=x_{i}-b y_{i}, \quad i=1,2, \ldots N, \\
& \dot{z}=\varepsilon k \sum_{i=1}^{N}\left(x_{i}-z\right)=\varepsilon k\left(\sum_{i=1}^{N} x_{i}-N z\right),
\end{aligned}
$$

describes the controlled array. Formally, for the uncontrolled array $C_{0} \rightarrow 0$, then $\varepsilon \rightarrow \infty$ and $z \rightarrow\left\langle x_{i}\right\rangle$. Consequently, (4) coincides with (3), as expected.

\section{Numerical results}

Equations (4) have been integrated using the MATHEMATICA 9 software. The results are presented in Fig. 3. Typical neuronal spiking (Fig. 3a) is observed until the controller is applied to the array at $t=100$. Then, after short transient process the system becomes stabilized to some non-zero (negative) steady state. Note that the control signal (Fig. 3b) after the transient process vanishes, i.e. stabilization is maintained with zero force. However, this does not mean that the controller can be switched off. Actually, the control force is within the noise level.

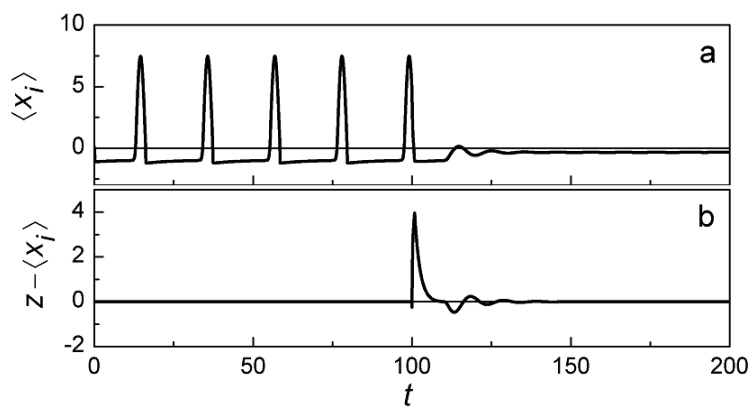

Fig. 3. Stabilizing unstable steady state in the array of three coupled FHN oscillators. (a) mean-field variable $\left\langle x_{i}\right\rangle$, (b) control signal $z-\left\langle x_{i}\right\rangle$. Controller is switched on at $t=100 . \quad N=3, a=4, b=0.1$, $c_{1}=-3.4, c_{2}=-3.2, c_{3}=-3.0, d_{1}=70, d_{2} 4, k=5$, $\varepsilon=0.04$.

\section{Experimental results}

Experiments have been performed using an array, composed of three mean-field coupled electronic FHN type oscillators, as shown in Fig. 1. The snapshots of the experimental signals are presented in Fig. 4. The stabilization experiments, shown in Fig. 4b, are in a very good agreement with the numerical simulations, presented in Fig. 3.

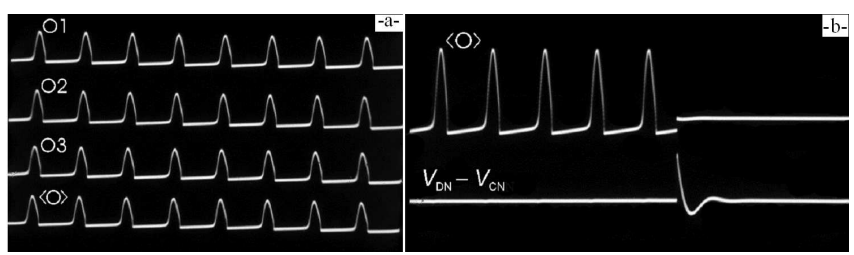

Fig. 4. (a) Outputs (voltages across capacitors C) from the synchronized oscillators O1, O2, O3 and mean-field voltage $\langle\mathrm{O}\rangle$ at the coupling node $\mathrm{CN} . R *=470 \Omega$. (b) Stabilization of steady state in the array of coupled oscillators; mean-field voltage $\langle\mathrm{O}\rangle$, and control signal $V_{\mathrm{DN}}-V_{\mathrm{CN}} . C_{0}=2.2 \mu \mathrm{F}, R_{1}=R_{2}=1 \mathrm{k} \Omega$, and $R_{3} \approx R_{\mathrm{g}}=1 \mathrm{k} \Omega$. Spike height $\approx 2 \mathrm{~V}$, period $\approx 0.7 \mathrm{~ms}$.

\section{Conclusions}

Concerning practical application of the described controller to real neuronal systems, we point out that the same electrode setup, as in the conventional DBS for the Parkinson disease treatment [12, 13], can be used. The electrodes implanted in either globus pallidus or subthalamic nucleus of the brain can be readily exploited. The pulse generator, used for the DBS, should be replaced with the feedback circuit FC. An important advantage of the proposed technique over the DBS is that the control signals, sent into the brain from the FC, are vanishing. 
In the recent papers $[14,15]$ detailed investigations, both analytical and numerical, have been carried out towards understanding of the mechanism of the existing DBS therapy technique. We hope that search for alternative methods, in particular using experimental approach, can contribute to the problem of the treatment of the Parkinson disease.

In addition, investigation of the possibility to control larger neuronal arrays, e.g. with $N=30$, described in [16], would be important.

\section{References}

[1] A. Pikovsky, M. Rosenblum, J. Kurths, Synchronization: A Universal Concept in Nonlinear Sciences, Cambridge University Press, Cambridge 2003.

[2] M.G. Rosenblum, A.S. Pikovsky, Phys. Rev. Lett. 92, 114102 (2004).

[3] O.V. Popovych, C. Hauptmann, P. Tass, Phys. Rev. Lett. 94, 164102 (2005).

[4] L.S. Tsimring, N.F. Rulkov, M.L. Larsen, M. Gabbay, Phys. Rev. Lett. 95, 014101 (2005).

[5] O.V. Popovych, C. Hauptmann, P. Tass, Biol. Cybern. 95, 69 (2006).
[6] P.A. Tass, Phase Resetting in Medicine and Biology, Springer, Berlin 2007.

[7] N. Tukhlina, M. Rosenblum, A. Pikovsky, J. Kurths, Phys. Rev. E 75, 011918 (2007).

[8] K. Pyragas, O.V. Popovych, P.A. Tass, Europhys. Lett. 80, 40002 (2007).

[9] A. Tamaševičius, E. Tamaševičiūtè, G. Mykolaitis, Appl. Phys. Lett. 101, 223703 (2012).

[10] A. Tamaševičius, E. Tamaševičiūtè, G. Mykolaitis, S. Bumelienè, R. Kirvaitis, R. Stoop, Lecture Notes Comp. Sci. 5768, 618 (2009).

[11] R. FitzHugh, Biophys. J. 1, 445 (1961).

[12] S. Breit, J.B. Schulz, A.-L. Benabid, Cell Tissue Res. 318, 275 (2004).

[13] J.S. Perlmutter, J.W. Mink, Annu. Rev. Neurosci. 29, 229 (2006).

[14] K. Pyragas, V. Novičenko, P. Tass, Biol. Cybern. 107, 669 (2013).

[15] I. Ratas, K. Pyragas, Nonlin. Dyn. 67, 2899 (2012).

[16] E. Tamaševičiūtè, G. Mykolaitis, A. Tamaševičius, Nonlin. Anal. Model. Control 17, 118 (2012). 